\title{
Influence of thermophoresis on MHD micropolar fluid over a moved permeable plate
}

\author{
R. Alouaoui*, M. N. Bouaziz** \\ *Biomaterials and Transport Phenomena Laboratory, University Medea, Algeria, 26000, E-mail: benkired@yahoo.fr \\ **Biomaterials and Transport Phenomena Laboratory, University Medea, Algeria, 26000, \\ E-mail:mn_bouaziz@email.com
}

cross $^{\text {ref }}$ http://dx.doi.org/10.5755/j01.mech.23.3.14723

\section{Introduction}

Local rotary inertia and couple stress are the effects encountered in polymeric fluids, exotic lubricants and liquid crystals and the hypothesis of a Newtonian fluid is unsuitable to describe the behaviour of these typical fluids. Later, they are termed micropolarity (Eringen, 1966), in the nomenclature of the rheology as a class of fluid micro-continua, Lukaszewicz[1].

The micropolar fluids are those which contain micro-constituents that can undergo rotation, the presence of which can affect the hydrodynamics of the flow. It has many practical applications, reported in the review articles and book [1].For example, plasma, liquid metal, ferro-fluid, colloidal fluids, polymeric fluids and blood as used largely become more and more important industrially. These fluids belong to the class of micropolar fluids and their applications are found in biomedical, engineering, in the dialysis of blood, in the transpiration cooling as well as in MHD power generators and cooling of nuclear reactor.

These kinds of the fluids have been modelled firstly by Eringen [2], adding a supplementary equation representing the local angular momentum which operates also in the classical momentum equation.

Micropolar fluids have been used by many authors in the boundary layer flow and heat transfer for different geometries under several physical and thermal conditions, for example [3-7].

The study of magnetic field effects on the flow, heat and mass transfer are relatively few when non-Newtonian fluid as the so-called micropolar fluid acts. Ishak et al. [8] analyse a micropolar fluid with MHD flow near a stagnation point. Thermal radiation and chemical reaction effects for the same type of fluid has been considered by Das [9].The first cited effect is also treated numerically by Mahmoud [10], considering a stretching surface with variable thermal conductivity. A numerical model is developed to examine the combined effects of Soret and Dufour on mixed convection magnetohydrodynamic heat and mass transfer in micropolar fluid-saturated Darcian porous medium in the presence of thermal radiation, non-uniform heat source/sink and Ohmic dissipation is presented by Pal and Chatterjee [11]. Seddeek et al. [12] derived an analytical solution for a magneto-micropolar fluid past a moving plate. Radiation, suction and blowing are the mechanisms incorporated. The steady laminar magnetohydrodynamic (MHD) boundary-layer flow past a wedge with constant surface heat flux immersed in an incompressible micropolar fluid in the presence of a variable magnetic field is investigated by
Ishak et al. [13]. Mahmoud and Waheed [14] examined an interaction between a MHD micropolar fluid and a moving surface with radiation. In the area of high temperature differences, Abd-El Aziz [15] studied the above effects on a moving semi-infinite plate with mixed convection flow of a micropolar fluid. The problem of the flow and heat transfer on a cylinder with a suction-case is discussed by Elbarbary and Elgazery [ 16]. Unsteady MHD natural convection is studied by Chamkha et al. [17] for a heated porous plate with combined effects.

Another interesting effect is the thermophoresis which cannot neglected under a strong thermal gradient. It is the mechanism known as a displacement of small particles under the presence of a thermal gradient causing a velocity called the thermophoresis velocity. This phenomenon has an important application in the industry and the environment. In this context, Alam et al. [18] presented the combined effect of joule heating and viscous dissipation and on a viscous incompressible fluid and over an inclined radiate isothermal permeable surface. For a vertical plate, Tsai and Huang [19] investigate the combined effects of thermophoresis and electrophoresis through a porous medium in the case of mixed convection flow. With the help of the Lie group analysis, Muhaimin et al. [20] studied the MHD free convective heat and mass transfer with thermophoresis and chemical reaction in the presence of heat source/sink. The influence of thermophoresis on MHD micropolar fluid flow is examined by Das [9].

A mass and heat transfer problem of a vertical permeable and the moving plate adjacent to micropolar fluid subject to a magnetic field with the thermophoresis effect is studied in the present paper. Motivated by the applications cited by Das [9] and considering the interesting case of moving the system, the present problem appears relatively new. The review of the published literature shows that the current problem has not yet received the attention of any researcher. All the mechanisms involved, namely the natural heat and mass transfer, the presence of a magnetic field and the velocity of the permeable plate necessitate a strong coupling and suggested an interesting behaviour to elucidate.

The resulting similarity solutions of the governing equations are obtained. Many results are presented and representative set is displayed graphically to illustrate the influence of the various dimensionless parameters. As such, it is in the main objective to determine the influence of the simultaneous aimed effects of heat and mass transfer from the moving permeable plate to the external medium, particularly for representative fluid microcontinua as the micropolar fluid. 


\section{Mathematical analysis}

Consider a steady laminar convective flow of an electrically conducting incompressible micropolar fluid along a semi-infinite vertical plate. A transverse magnetic field $B_{0}$ is applied normally to the plate in the presence of thermophoresis. As considered by Das [9], the magnetic field varies in strength as a function of $x$. In this formulation, the magnetic dissipation is not ignored. The plate is permeable and received/loss fluid with a transverse velocity $v_{w}$ and moves along $x$-direction with a constant velocity $U$. The wall is maintained at constant temperature and concentration, $T_{w}$ and $C_{w}$, and these values are assumed to be greater than the ambient temperature and concentration, $T_{\infty}$ and $C_{\infty}$, respectively. The $x$-direction which is taken along the plate is also the direction of the flow and $y$-axis is normal to it. The schematic diagram of the present physical problem is depicted in Fig. 1.

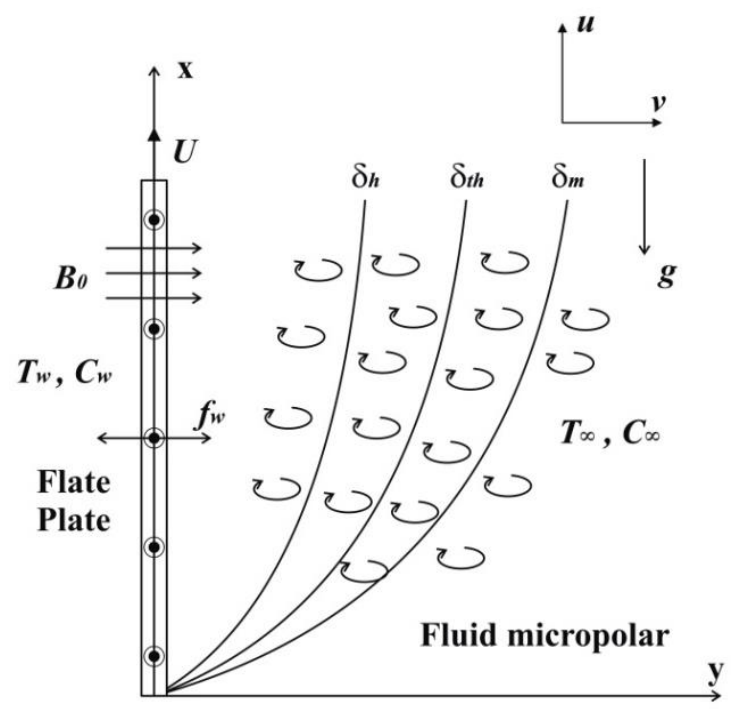

Fig. 1 Physical model and coordinate system

Then, under these assumptions with Boussinesq's approximation and regarding the model developed in the work of Das [9], the governing conservation equations of mass, momentum and angular momentum, energy and species concentration can be written as

$$
\begin{aligned}
& \frac{\partial u}{\partial x}+\frac{\partial v}{\partial y}=0 ; \\
& u \frac{\partial u}{\partial x}+v \frac{\partial v}{\partial y}=\left(\frac{\mu+\kappa}{\rho}\right) \frac{\partial^{2} u}{\partial y^{2}}+\frac{\kappa}{\rho} \frac{\partial N}{\partial y}+ \\
& +g \beta\left(T-T_{\infty}\right)+g \beta^{*}\left(C-C_{\infty}\right)-\frac{\sigma B_{0}^{2}}{\rho} u ; \\
& j \rho\left(u \frac{\partial N}{\partial x}+v \frac{\partial N}{\partial y}\right)=\gamma \frac{\partial^{2} N}{\partial y^{2}}-K\left(2 N+\frac{\partial u}{\partial y}\right) ; \\
& u \frac{\partial T}{\partial x}+v \frac{\partial T}{\partial y}=\frac{\lambda_{g}}{\rho c_{p}} \frac{\partial^{2} T}{\partial y^{2}}+\frac{\sigma B_{0}^{2}}{\rho c_{p}} u^{2} ; \\
& u \frac{\partial C}{\partial x}+v \frac{\partial C}{\partial y}=D \frac{\partial^{2} C}{\partial y^{2}}-\frac{\partial}{\partial y}\left(V_{T}\left(C-C_{\infty}\right)\right),
\end{aligned}
$$

here $T$ is the temperature; $C$ is the species concentration in the boundary layer and $N$ the component of microrotation vector normal to $x-y$ plane. Others parameters and quantities are specified in the nomenclature. We follow the work of many recent authors as the work of Long Hsiao [21], by assuming that the spin gradient viscosity is:

$$
\gamma=(\mu+\kappa / 2) j
$$

$V_{T}$ represents the thermophoresis velocity:

$$
V_{T}=k_{1}\left(\frac{v}{T_{r}} \frac{\partial T}{\partial y}\right)
$$

where $k_{1}$ is thermophoretic coefficient and $T_{r}$ is some reference temperature.

The corresponding permeable wall and stream boundary conditions are defined as follows:

$$
\left.\begin{array}{l}
\text { at } y=0: u=U ; v= \pm v_{w} ; T=T_{w} ; C=C_{w} ; N=-n \frac{\partial u}{\partial x} \\
\text { for } y->\infty: u=0 ; T->T_{\infty} ; C=C_{\infty} ; N->0 .
\end{array}\right\}
$$

In the above equation $n=1 / 2$ which represents a weak concentration of particle flows and indicates the vanishing of anti-symmetric part of the stress tensor, Ahmadi [22].

Following Yacob et al. [23], dimensionless quantities are introduced to obtain the similarity solutions:

$$
\eta=(U / v x)^{\frac{1}{2}} y ; \psi=(v x U)^{\frac{1}{2}} f(\eta) ; N=U(U / v x)^{\frac{1}{2}} h(\eta)
$$
centration:

While for the dimensionless temperature and con-

$$
\theta(\eta)=\frac{T-T_{\infty}}{T_{w}-T_{\infty}} ; \phi(\eta)=\frac{C-C_{\infty}}{C_{w}-C_{\infty}}
$$

where $\eta$ is the independent similarity variable; $f(\eta)$ is the dimensionless velocity function; $h(\eta)$ is the dimensionless microrotation; $\theta(\eta)$ is the dimensionless temperature and $\phi(\eta)$ is the dimensionless concentration. Further, $\psi$ is the stream function which is defined in the usual way as:

$$
u=\frac{\partial \psi}{\partial y} ; v=-\frac{\partial \psi}{\partial x} .
$$

Then, the velocity components become:

$$
u=U f^{\prime}(\eta) \text { and } v=\frac{1}{2}(v U / x)^{\frac{1}{2}}\left(\eta f^{\prime}-f\right) .
$$

Using the non-dimensional quantities, the equations (1-5) with the boundary conditions (6) can be further reduced to a set of ordinary differential equations for which numerical solutions will be more easily obtained:

$$
(1+K) f^{\prime \prime \prime}+\frac{1}{2} f f^{\prime \prime}-M f^{\prime}+K h^{\prime}+G r_{x} \theta+G m_{x} \phi=0
$$




$$
\begin{aligned}
& \left(1+\frac{K}{2}\right) h^{\prime \prime}-K\left(2 h+f^{\prime \prime}\right)+\frac{1}{2}(h f)^{\prime}=0 ; \\
& \theta^{\prime \prime}+\frac{1}{2} \operatorname{Prf} \theta^{\prime}+M E c P r f^{\prime 2}=0 ; \\
& \phi^{\prime \prime}+\frac{1}{2} S c f \phi^{\prime}+S c \tau\left(\theta^{\prime \prime} \phi+\theta^{\prime} \phi^{\prime}\right)=0 .
\end{aligned}
$$

The boundary conditions become:

$$
\begin{aligned}
& \text { at } \eta=0: f^{\prime}(0)=1 ; f(0)=f_{w} ; h(0)=-\frac{1}{2} f^{\prime \prime}(0) ; \\
& \theta(0)=\phi(0)=1, \\
& \text { for } \eta->\infty: f^{\prime}(\infty)=0 ; h(\infty)->\theta(\infty)-> \\
& ->\phi(\infty)->0 ; j=v x / U
\end{aligned}
$$

where, we have taken $j=v x / U$ as the microinertia density.

$$
f_{w}=-2 v_{w} \sqrt{x / v U} \text { represents suction/injection ve- }
$$

locity at the plate.

The various parameters involved are defined by:

$$
\begin{aligned}
& G r_{x}=\frac{g \beta\left(T_{w}-T_{\infty}\right) x}{U^{2}} ; G m_{x}=\frac{g \beta^{*}\left(C_{w}-C_{\infty}\right) x}{U^{2}} ; K=\frac{\kappa}{\mu} ; \\
& M=\frac{H a_{x}^{2}}{R e_{x}}=\frac{\sigma B_{0}^{2} x}{\rho U} ; \quad E c=\frac{U^{2}}{c_{p}\left(T_{w}-T_{\infty}\right)} ; \operatorname{Pr}=\frac{v}{\alpha} ; \\
& \tau=-\frac{k_{1}\left(T_{w}-T_{\infty}\right)}{T_{r}} ; S c=\frac{v}{D},
\end{aligned}
$$

where $G r_{x}, G m_{x}, K, M, E c, P r, \tau$ denote the local thermal Grashof number, local mass Grashof number, the material parameter, the magnetic parameter, the Eckert number, the Prandtl number and the thermophoretic parameter respectively, while $S c$ is the Schmidt number. $H a_{x}$ and $R e_{x}$ linked to $M$ are the known Hartman and Reynolds numbers, respectively.

The surface shear stress $t_{w}$, the wall couple stress $m_{w}$, the surface heat flux $q_{w}$ and the surface mass flux $q^{\prime}{ }_{w}$ are given by:

$$
\begin{aligned}
& \tau_{w}=\left[(\mu+\kappa) \frac{\partial u}{\partial x}+\kappa N\right]_{y=0} ; m_{w}=\gamma\left(\frac{\partial N}{\partial y}\right)_{y=0} ; \\
& q_{w}=-\lambda_{g}\left(\frac{\partial T}{\partial y}\right)_{y=0} ; q_{w}{ }^{\prime}=-D\left(\frac{\partial C}{\partial y}\right)_{y=0} .
\end{aligned}
$$

For practical applications, the corresponding physical quantities of interest are the skin friction coefficient $C_{f}$, the dimensionless wall couple stress $M_{w}$, the local Nusselt number $N u_{x}$ and the local Sherwood number $S h_{x}$, which are defined as:

$$
C_{f}=\frac{2 \tau_{w}}{\rho U^{2}} ; M_{w}=\frac{m_{w}}{\rho U^{2} x} ; N u_{x}=\frac{x q_{w}}{\lambda_{g}\left(T_{w}-T_{\infty}\right)} ;
$$

$$
S h_{x}=\frac{x q_{w}{ }^{\prime}}{D\left(C_{w}-C_{\infty}\right)} .
$$

Using the dimensionless variables, we obtain:

$$
\left.\begin{array}{l}
\frac{1}{2} C_{f} R e_{x}^{\frac{1}{2}}=\left(1+\frac{K}{2}\right) f^{\prime \prime}(0) ; M_{w} R e_{x}=\left(1+\frac{K}{2}\right) h^{\prime}(0) \\
\frac{N u_{x}}{\operatorname{Re}_{x}^{\frac{1}{2}}}=-\theta^{\prime}(0) ; \frac{S h_{x}}{\operatorname{Re}_{x}^{\frac{1}{2}}}=-\phi^{\prime}(0) .
\end{array}\right\}
$$

\section{Results and discussion}

The set of the coupled ordinary differential Eq. (7)(10) is highly nonlinear and cannot be solved analytically. Together with the boundary conditions (11), they form a two point boundary value problem, which can be solved using the finite difference method that implements the 3-stage Lobatto collocation formula. A continuous solution is provided by the collocation polynomial that is accurate up the fourthorder. Mesh selection and error control are linked with the residual of the continuous solution. Subintervals are generated to proceed of integration.

The flow regions are controlled by thermophysical parameters, namely $G r_{x}, G m_{x}, K, \tau, M, E c$, Prandtl $S c$. Numerical computations are carried out for different values of the parameters shown in all figures. To check the accuracy of the code used, preliminary calculations are executed on the basis of the values implemented by Alam et al. [20], and by taking into account the specific differences in the transformed variables. From the Fig. 2 dressed with $P r=0.7$; $S c=1000$ and $M=0 ; E c=G r_{x}, G m_{x}=0 ; K=0 ; \tau=1$, the velocity predicted for comparison are in the good agreement.

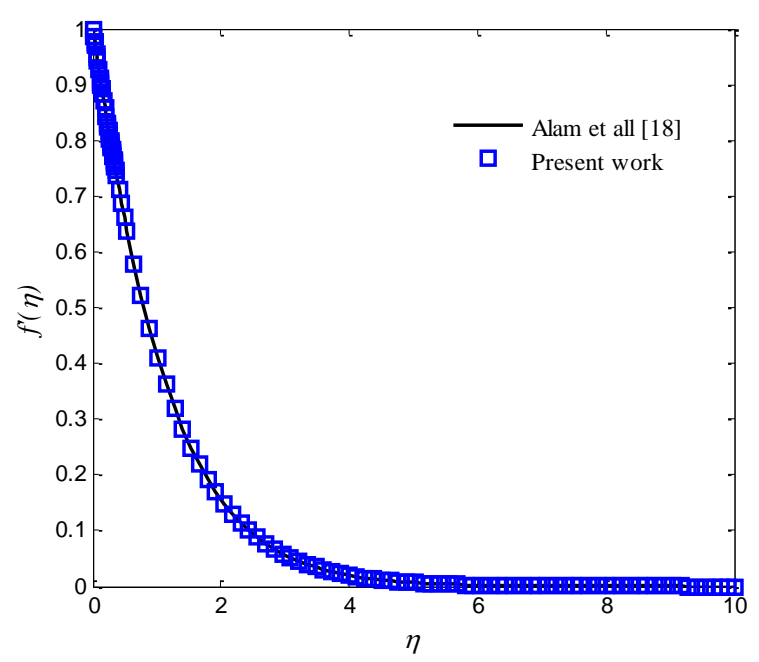

Fig. 2 Velocity profiles $f^{\prime}(\eta)$ for comparison with results of Alam et al. [18]

Next, numerical computations have been carried out for the velocity, microrotation, temperature and concentration profiles. To compare the behaviour of the strongly and weak micropolar fluid, the main results in all figures are displayed graphically for $K=0.1$ and $K=6$.

A reference case is adopted with these parameters, $\operatorname{Pr}=0.71 ; S c=0.3$ and $E c=0.1$; while the local thermal 
Grashof number is 2.0 and the local concentration Grashof number is chosen to be 0.5 . The values of strength of the magnetic field are chosen 0.5 and 1.0 whereas $M=0.0$ corresponding to the flow without the magnetic field. Similarly, the thermophoretic parameter $\tau$ takes 1.0 and 2.0 whereas $\tau=0$ represents the absence of the thermophoresis particle deposition. To illustrate the suction/injection flow through the permeable plate, the values $f_{w}=+1$ corresponds to the suction-case and $f_{w}=-1$ is chosen for the injection-case. The impermeable-case is represented naturally by $f_{w}=0$.

\subsection{Effect of the magnetic parameter $M$ and the material pa- rameter $K$}

Typical curves are obtained for the velocity of the fluid, Fig. 3. From this figure, it can observe that increasing the magnetic parameter $M$ decreases the velocity $f$ ' inside the hydrodynamic boundary layer. This is due to the Lorentz force which acts and decelerates the motion of the fluid. The useful comparison between a weak and strongly micropolar fluids shows that this magnetic effect is more important for a true micropolar fluid. The magnetic field develops the Lorentz force which perturbs not only the main flow but also the microrotation of the particles.

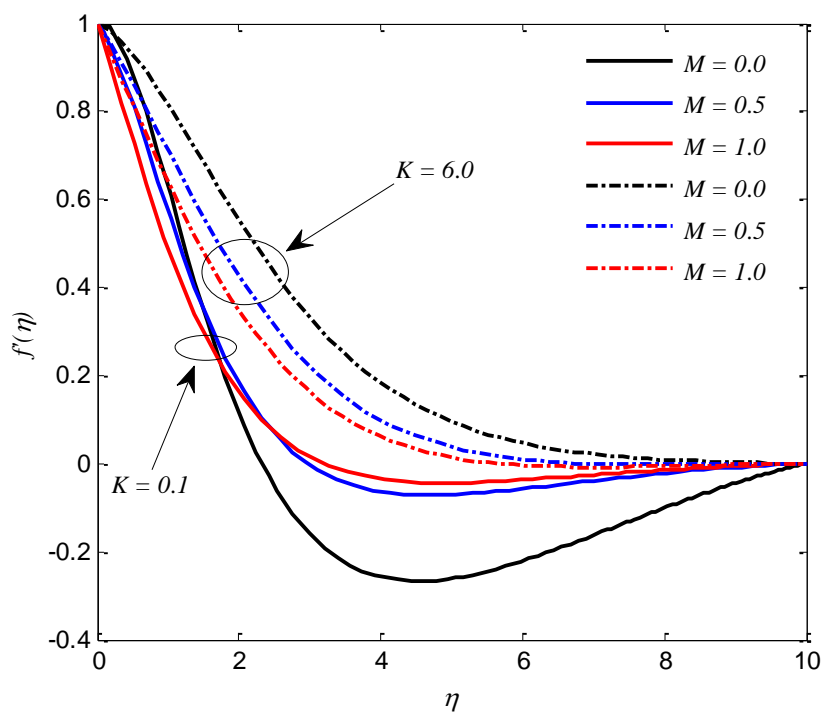

Fig. 3 Velocity profiles $f^{\prime}(\eta)$ with $M\left(G r_{x}=2.0, G m_{x}=0.5\right.$, $\left.\operatorname{Pr}=0.71, f_{w}=1.0, E c=0.1, S c=0.3, \tau=1.0\right)$

Fig. 4 depicts the angular velocity profile. As it can see, the effect of $M$ on the micropolar fluid is more pronounced neat the moving plate, where the increase of the magnetic field corresponds to a substantial increase of the angular velocity. At a representative zone $\eta>2$, less effect is observed. Although the same effect encounters in the same zone for a weakly micropolar fluid, the tendency is such that the angular velocity falls more dramatically with $M$. This is explained by the difference in concentration of the particles and in the two cases.

It noted from Fig. 5 that applying a magnetic field to the flow leads to an increase of the temperature profile with the increase of $M$. This is attributed to the reduced velocity, as commented above. Then, the heat exchanged with the moving plate is reduced, causing in turn an in creased temperature in the thermal boundary layer. Comparatively, the corresponding profiles a weakly micropolar fluid exhibit a greater temperature and a thicker boundary layer. As a whole, this indicates that extensive heat can be obtained by a micropolar fluid rather than a classical fluid.

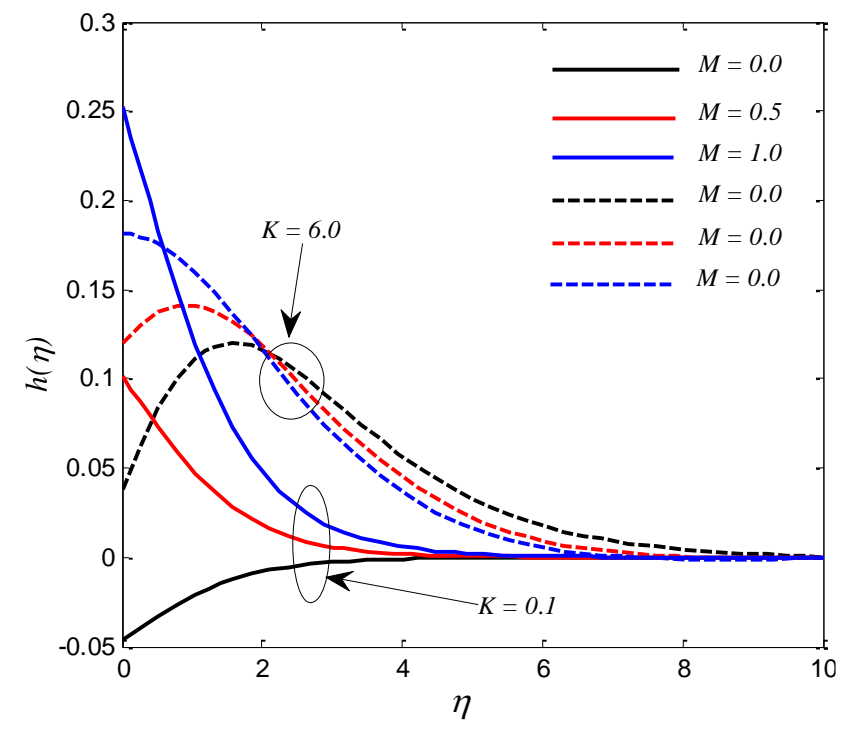

Fig. 4 Microrotation profiles $h(\eta)$ with $M\left(G r_{x}=2.0\right.$, $G m_{x}=0.5, \operatorname{Pr}=0.71, f_{w}=1.0, E c=0.1, S c=0.3$, $\tau=1.0)$

Fig. 6 displays the concentration profiles under the effect of $M$ here again the use of a micropolar fluid is an interesting way to enhance the mass transfer. For the present conditions experienced and a classical fluid, it is worth mentioning that the temperature profiles are insensible to change of $M$. Like the temperature inside the thermal boundary layer, it can also see from this figure that the concentration of the fluid in the similar mass boundary layer increases as $M$ increases. The ticker boundary layer is synonymous of slow mass exchange, caused by the retarded velocity or the presence of the Lorentz force.

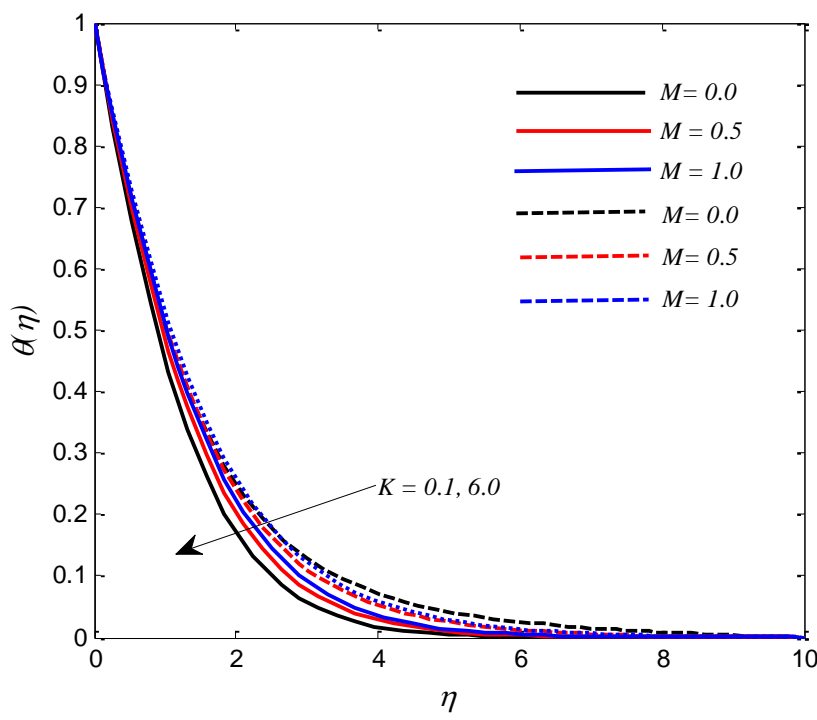

Fig. 5 Temperature profiles $\theta(\eta)$ with $M\left(G r_{x}=2.0\right.$, $G m_{x}=0.5, \operatorname{Pr}=0.71, f_{w}=1.0, E c=0.1, S c=0.3$, $\tau=1.0)$ 


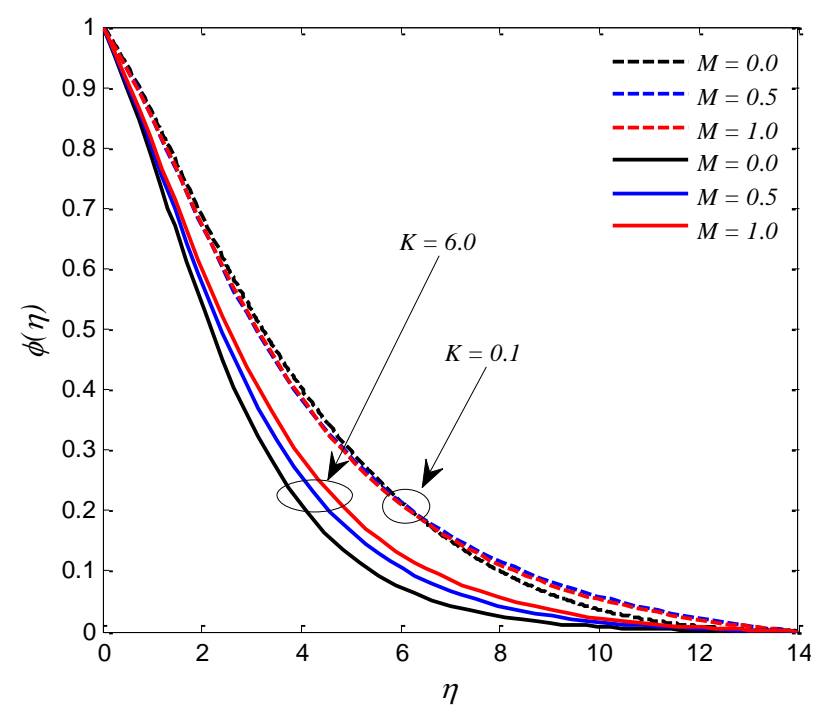

Fig. 6 Concentration profiles $\phi(\eta)$ with $M\left(G r_{x}=2.0\right.$, $G m_{x}=0.5, \operatorname{Pr}=0.71, f_{w}=1.0, E c=0.1, S c=0.3$, $\tau=1.0)$

3.2. Effect of the thermophoresis parameter $\tau$ and the material parameter $K$

The thermophoresis phenomenon or the migration of the particles caused by the presence of a thermal gradient constitutes an additional mechanism to the diffusion of the species. The velocity is reduced uniformly when the parameter $\tau$ increase, Fig. 7. For a strong micropolarity of the fluid, this effect augments the velocity of the fluid and the momentum boundary layer is naturally augmented also. As comprehensive and physical sense, it is evident that the micropolar fluid can be considered more viscous than a classical fluid. The vortex viscosity plays an important role relative to the flow. In the vicinity of the moving plate, the effect of $\tau$ on the microrotation of the particles, as shown in Fig. 8, is not negligible. It is observed that the maximal departure is located on the plate and the angular velocity is greater as $\tau$ increase. On the other hand, the microrotation is concentrated at the wall and fall abruptly in relation to the weak micropolarity. Probably, the vortex viscosity as the viscosity operates essentially at the wall-fluid interface.

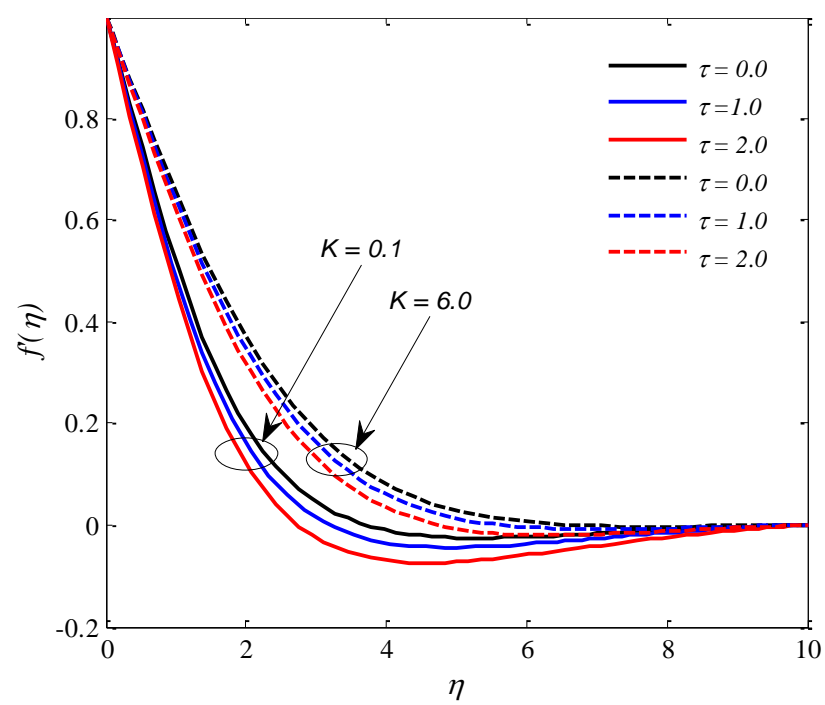

Fig. 7 Velocity profiles $f^{\prime}(\eta)$ with $\tau\left(G r_{x}=2.0, G m_{x}=0.5\right.$, $\left.\operatorname{Pr}=0.71, f_{w}=1.0, \quad E c=0.1, \quad S c=0.3, \quad M=1.0\right)$

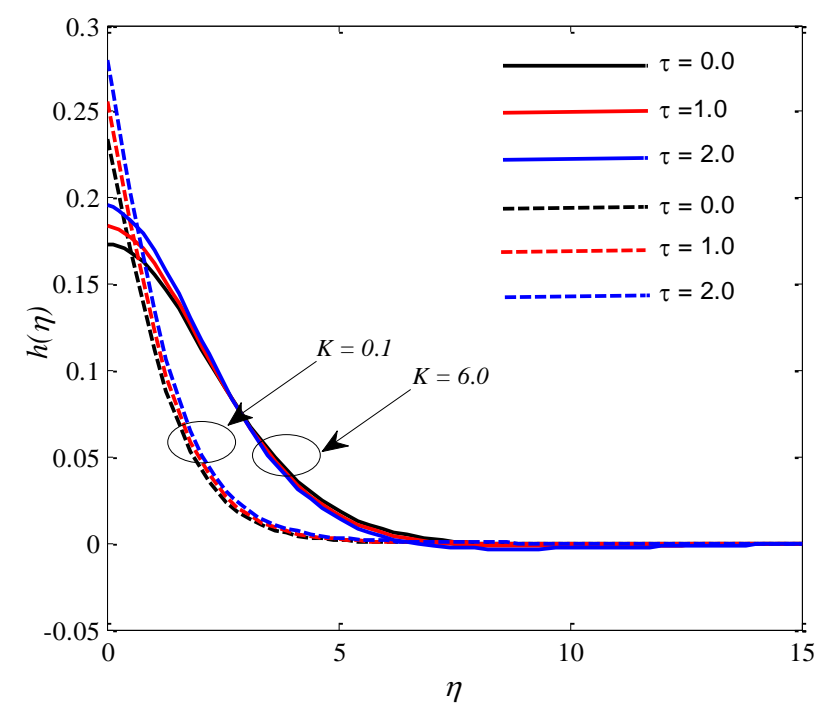

Fig. 8 Microrotation profiles $h(\eta)$ with $\tau \quad\left(G r_{x}=2.0\right.$, $G m_{x}=0.5, \operatorname{Pr}=0.71, f_{w}=1.0, E c=0.1, S c=0.3$, $M=1.0$ )

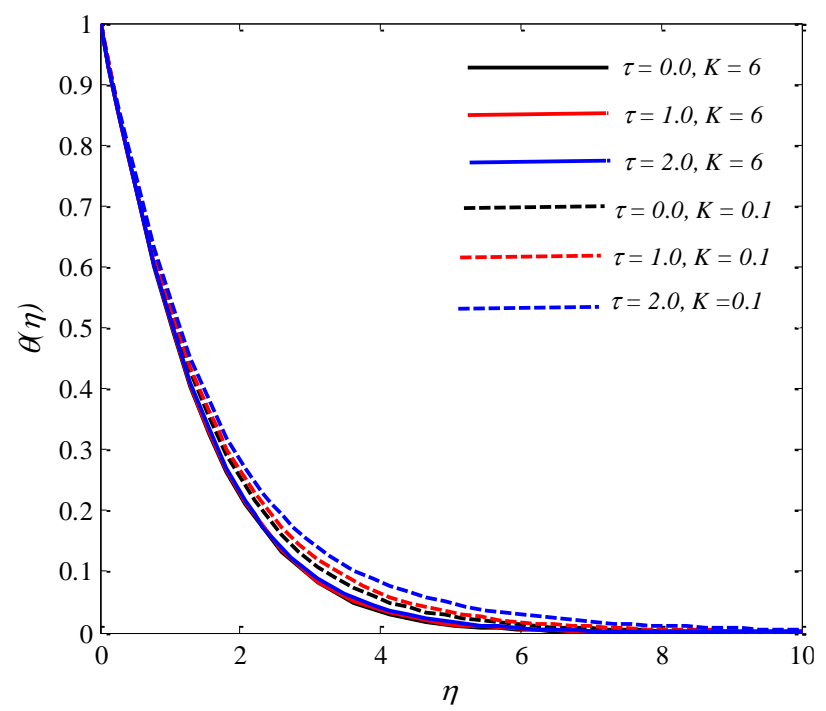

Fig. 9 Temperature profiles $\theta(\eta)$ with $\tau \quad\left(G r_{x}=2.0\right.$, $G m_{x}=0.5, \operatorname{Pr}=0.71, f_{w}=1.0, E c=0.1, S c=0.3$, $M=1.0)$

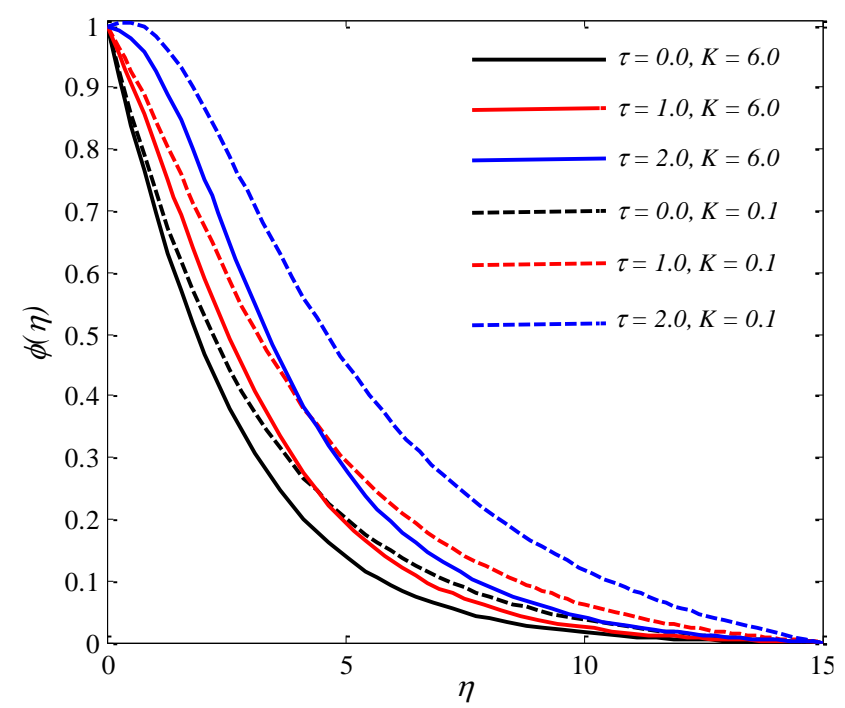

Fig. 10 Concentration profiles $\phi(\eta)$ with $\tau\left(G r_{x}=2.0\right.$, $G m_{x}=0.5, \operatorname{Pr}=0.71, f_{w}=1.0, E c=0.1, S c=0.3$, $M=1.0)$ 
From Fig. 9, the temperature profiles are not affected by the thermophoresis effect. This can be explained by the fact that the present phenomenon is a mass driven mechanism. Inversely, in Fig. 10, the increasing of the thermphoretic parameter $\tau$ causes an increase of the concentration of the fluid. Superposed to the basic mechanism, the mass boundary layer is magnified by the presence of the thermophoresis. It can state that improved mass transfer is obtained by the use of a micropolar fluid.

\subsection{Effect of the suction/injection parameter $f_{w}$ and the ma- terial parameter $K$}

The effects of suction/injection in conjunction with a micropolar fluid are depicted in Figs. 11-14. The passage of suction to injection leads to enhance the hydrodynamic boundary layer, as reported in Fig. 11. It is evident that the velocity of the fluid decreases when suction occurs and viceversa. Conversely, the thickness of the hydrodynamic boundary layer is less in the suction case than in the injection case. The results show that the micropolar fluid presents more resistance to flow, as expected. However, this effect can be reversed in the injection case with a strong micropolar fluid. Fig. 12 displays the opposite tendency for the microrotation, particularly in the first region near the moving permeable plate. It should be noted that the angular velocity starts from a minimum value to reach a peak value and then starts decreasing. Such behavior is not appearing for a micropolar fluid in the suction case. It is interesting to note that a negative value of the angular velocity is observed for a weak micropolar fluid in the injection case. These results demonstrate that the microrotation is intensive in the middle of the boundary layer, depending to $K$ and $f_{w}$. The influence of the suction/injection parameter $f_{w}$ on the temperature profile is shown in Fig. 13. In the suction case the temperature decreases while opposite effect is seen in the injection case. Hence, slight improvement of the heat transfer with the micropolar fluid is encountered in these conditions. A similar trend is remarked from the concentration profile, as plotted in Fig. 14.

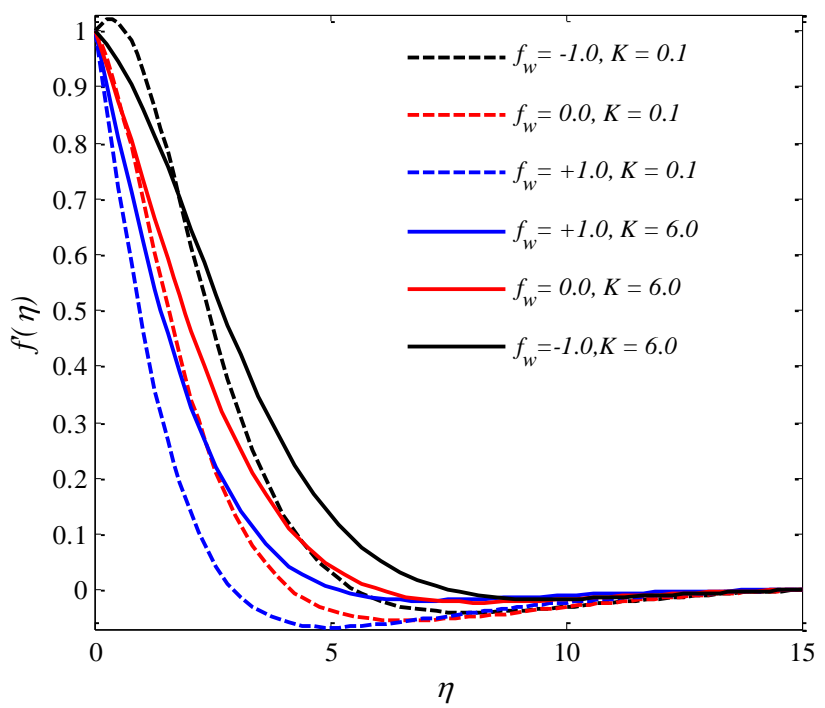

Fig. 11 Velocity profiles $f^{\prime}(\eta)$ with $f_{w}\left(G r_{x}=2.0, G m_{x}=0.5\right.$, $\operatorname{Pr}=0.71, \quad M=1.0, \quad E c=0.1, \quad S c=0.3, \quad \tau=1.0$ )

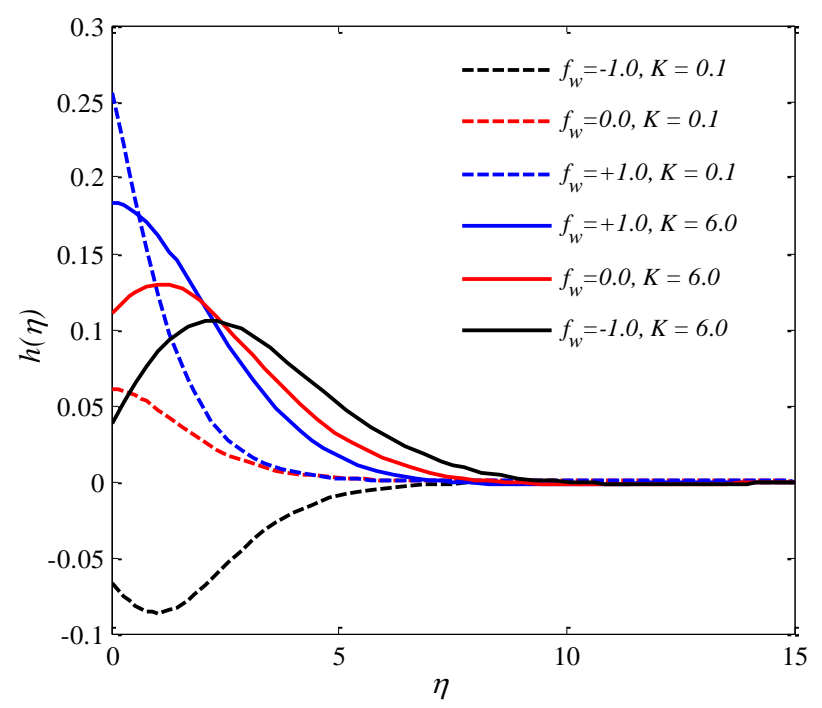

Fig. 12 Microrotation profiles $h(\eta)$ with $f_{w} \quad\left(G r_{x}=2.0\right.$, $G m_{x}=0.5, P r=0.71, M=1.0, E c=0.1, S c=0.3$, $\tau=1.0)$

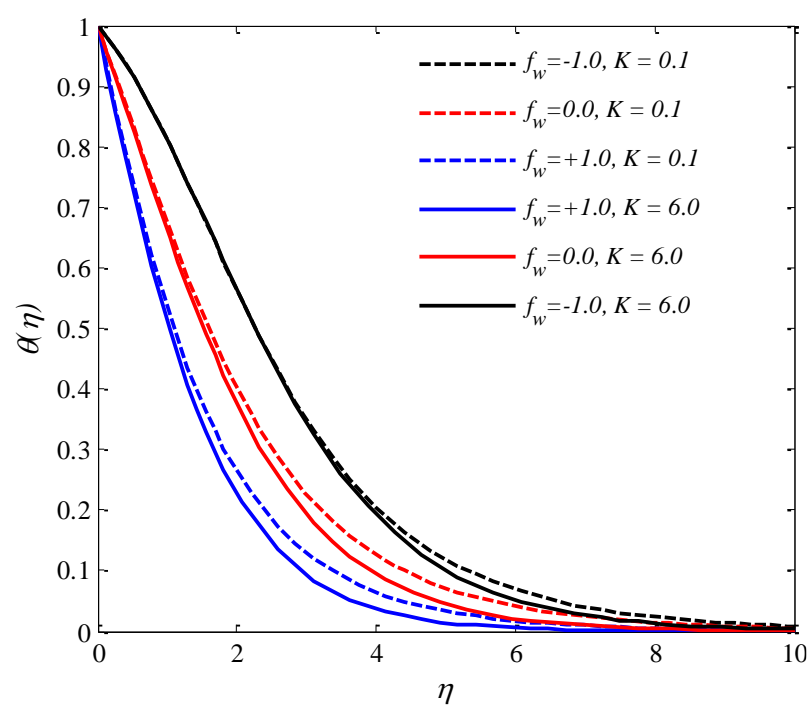

Fig. 13 Temperature profiles $\theta(\eta)$ with $f_{w} \quad\left(G r_{x}=2.0\right.$, $G m_{x}=0.5, \operatorname{Pr}=0.71, M=1.0, E c=0.1, S c=0.3$, $\tau=1.0)$

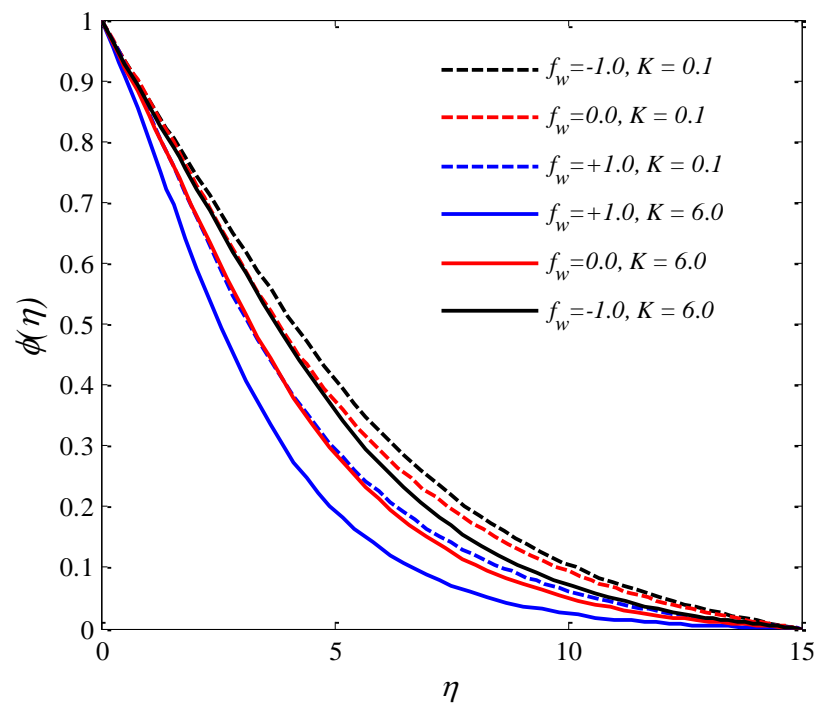

Fig. 14 Concentration profiles $\phi(\eta)$ with $f_{w} \quad\left(G r_{x}=2.0\right.$, $G m_{x}=0.5, \operatorname{Pr}=0.71, M=1.0, E c=0.1, S c=0.3$, $\tau=1.0)$ 
3.4. Variations of the skin friction coefficient, the wall couple stress, the local Nusselt and Sherwood numbers

Now, we are concerned to the variations of the skin friction coefficient, the local Nusselt and Sherwood numbers for different values of local magnetic parameter $M$, the material parameter $K$, thermophoretic parameter $\tau$, the Suction/injection parameter $f_{w}$ and $\operatorname{Pr}$ that are illustrated in the Tables 1 and 2 . It can be seen from Table 1 that the magnetic field affects strongly the local skin friction coefficient and weakly the other quantities. On the other hand, the mass transfer is sensitive to the effect of the thermophoresis and much less for the heat transfer.

From Table 2, only the suction case has a strong effect on the local wall couple stress as well as the local skin-friction coefficient, whereas the local Nusselt number is slightly affected and the local Sherwood number remains insensitive to the three cases studied. In all the cases, it can be noticed that the skin-friction is concerned mainly with the variations of the micropolarity. The wall couple stress is significantly varied with the weak values of the material parameter.

Table 1

Values of $\frac{1}{2} C_{f} R e_{x}^{\frac{1}{2}}, M_{w} R e_{x}, N u_{x} R e_{x}^{-\frac{1}{2}}, S h_{x} R e_{x}^{-\frac{1}{2}}$ and for selected values of

$\tau, M$ and $K$ with $G r_{x}=2.0, G m_{x}=0.5, P r=0.71, f_{w}=1.0, E c=0.1, S c=0.3$

\begin{tabular}{|c|c|c|c|c|c|c|}
\hline$M$ & $\tau$ & $K$ & $\frac{1}{2} C_{f} R e_{x}^{\frac{1}{2}}$ & $M_{w} R e_{x}$ & $N u_{x} R e_{x}^{-\frac{1}{2}}$ & $S h_{x} R e_{x}^{-\frac{1}{2}}$ \\
\hline \multirow{3}{*}{0.0} & \multirow{3}{*}{1.0} & 0.1 & -0.0697 & 0.1149 & 0.6197 & 0.1241 \\
\hline & & 3.0 & -0.1387 & 0.3757 & 0.6710 & 0.1730 \\
\hline & & 6 & -0.3020 & 0.3900 & 0.6829 & 0.1875 \\
\hline \multirow{3}{*}{1.0} & \multirow{3}{*}{1.0} & 0.1 & -0.5297 & -0.0969 & 0.5589 & 0.1499 \\
\hline & & 3.0 & -1.0470 & -0.0190 & 0.5754 & 0.1659 \\
\hline & & 6 & -1.4532 & -0.0164 & 0.5829 & 0.1781 \\
\hline \multirow{3}{*}{2.0} & \multirow{3}{*}{1.0} & 0.1 & -1.0157 & -0.2634 & 0.5059 & 0.1513 \\
\hline & & 3.0 & -1.6777 & -0.3290 & 0.5169 & 0.1649 \\
\hline & & 6 & -2.2232 & -0.3488 & 0.5214 & 0.1766 \\
\hline \multirow{9}{*}{1.0} & \multirow{3}{*}{0.0} & 0.1 & -0.4881 & -0.0840 & 0.5652 & 0.3012 \\
\hline & & 3.0 & -0.9822 & -0.0042 & 0.5787 & 0.3186 \\
\hline & & 6 & -1.3752 & -0.0016 & 0.5847 & 0.3303 \\
\hline & \multirow{3}{*}{1.0} & 0.1 & -0.529 & -0.0969 & 0.5589 & 0.1499 \\
\hline & & 3.0 & -1.0470 & -0.0190 & 0.5754 & 0.1659 \\
\hline & & 6 & -1.4532 & -0.0164 & 0.5829 & 0.1781 \\
\hline & \multirow{3}{*}{2.0} & 0.1 & -0.5759 & -0.1108 & 0.5495 & 0.0043 \\
\hline & & 3.0 & -1.1235 & -0.0350 & 0.5704 & 0.0172 \\
\hline & & 6 & -1.5468 & -0.0328 & 0.5803 & 0.0296 \\
\hline
\end{tabular}

Table 2

Values of $\frac{1}{2} C_{f} R e_{x}^{\frac{1}{2}}, M_{w} R e_{x}, N u_{x} R e_{x}^{-\frac{1}{2}}, S h_{x} R e_{x}^{-\frac{1}{2}}$ and for selected values of

$\tau, f_{w}$ and $K$ with $G r_{x}=2.0, G m_{x}=0.5, P r=0.71, M=1.0, E c=0.1, S c=0.3$

\begin{tabular}{|c|c|c|c|c|c|c|}
\hline$\tau$ & $f_{w}$ & $K$ & $\frac{1}{2} C_{f} R e_{x}^{\frac{1}{2}}$ & $M_{w} R e_{x}$ & $N u_{x} R e_{x}^{-\frac{1}{2}}$ & $S h_{x} R e_{x}^{-\frac{1}{2}}$ \\
\hline \multirow{3}{*}{0.0} & \multirow{3}{*}{-1.0} & 0.1 & -0.1261 & 0.0464 & 0.1368 & 0.1519 \\
\hline & & 3.0 & -0.0710 & 0.1815 & 0.1376 & 0.1527 \\
\hline & & 6 & -0.2960 & 0.2016 & 0.1383 & 0.1545 \\
\hline \multirow{3}{*}{1.0} & \multirow{3}{*}{0.0} & 0.1 & -0.1363 & 0.0483 & 0.3184 & 0.1517 \\
\hline & & 3.0 & -0.5242 & 0.1312 & 0.3252 & 0.1594 \\
\hline & & 6 & -0.8652 & 0.1304 & 0.3293 & 0.1660 \\
\hline \multirow{3}{*}{2.0} & \multirow{3}{*}{+1.0} & 0.1 & -0.5297 & -0.0969 & 0.5589 & 0.1499 \\
\hline & & 3.0 & -1.0470 & -0.0190 & 0.5754 & 0.1659 \\
\hline & & 6 & -1.4532 & -0.0164 & 0.5829 & 0.1781 \\
\hline
\end{tabular}

\section{Conclusion}

The effects of magnetic field and thermophoresis effect on steady laminar boundary layer flow of an incompressible electrically conducting micropolar fluid over a permeable vertical moving plate are studied. The similarity transformations are involved and the governing system of non linear partial differential equations is converted into a set of non linear ordinary differential equations. Numerical results are presented and we can conclude the following 
from our investigation:

- The magnetic parameter has a strong effect on the characteristics of the micropolar fluid flow. It plays other dissipation effect which can affect substantially the multiple exchanges of the mass and energy transfers.

- Working with high micropolar leads to modifying the temperature, angular velocity and species mass profiles, which in turn affect the rates of heat and mass, but the effect is more perceptible for the flow velocity, and greater influence is observed for the skin friction coefficient.

- It is found that the rate of mass transfer decreases strongly with increasing the thermophoretic parameter.

- It is clear that the suction/injection of the permeable plate can be modified the flow, angular velocity and dimensionless temperature and mass. For a point of view of control, the skin friction, wall couple stress, heat and mass transfer rate can be regulated principally by a permeable plate in the suction case.

\section{Acknowledgments}

This work was supported in entire part by the Biomaterials and transport phenomena laboratory agreement $\mathrm{N}^{\circ}$ 303 03-12-2003, at university of Medea. R. Alouaoui and M.N. Bouaziz acknowledge the financial support provided by DG-RSDT of Algeria.

\section{References}

1. Lukaszewicz, G. 1999. Micropolar Fluids. Theory and Applications, Springer Science\& Business Media, LLC, $253 \mathrm{p}$.

Available from internet (e-book): http://link.springer. com/book10.1007/978-1-4612-0641-5.

2. Eringen, A.C. 1999. Microcontinuum Field Theories. Foundations and Solids, Springer Science \& Business Media, LLC, 325 p.

http://cds.cern.ch/record/200638210.1007/978-1-46120555-5.

3. Gorla, R.S.R.; Nakamura, S.1995. Mixed convection of a micropolar fluid from a rotating cone, International Journal Heat Fluid Flow 16: 69-73.

Available from internet: http://www.sciencedirect.com/ science/article/pii/ 0142727X94000092.

4. Lok, Y.Y.; Amin, N.; Pop I. 2006. Unsteady mixed convection flow of a micropolar fluid near the stagnation point on a vertical surface, International Journal of Thermal Sciences 45: 1149-1157.

http://dx.doi.org/10.1016/j.ijthermalsci.2006.01.015.

5. Aydın, O.; Pop I. 2007. Natural convection in a differentially heated enclosure filled with a micropolar fluid. International Journal Thermal Sciences 46: 963-969. http://dx.doi.org/10.1016/j.ijthermalsci.2006.11.018.

6. Ishak, A.; Nazar, R.; Pop, I. 2008. Stagnation flow of a micropolar fluid towards a vertical permeable surface, International Communications Heat Mass Transfer 35: 276-281.

http://dx.doi.org/10.1016/j.icheatmasstransfer.2007.07.007.

7. Ishak, A.; Nazar, R.; Pop, I. 2009. Dual solutions in mixed convection boundary layer flow of micropolar fluids, Communications Nonlinear Science Numerical Simulation 14: 1324-1333. http://dx.doi.org/10.1016/j.cnsns.2008.01.017.

8. Ishak, A.; Nazar, R.; Pop, I. 2008. Magnetohydrodynamic (MHD) flow of a micropolar fluid towards a stagnation point on a vertical surface, Computational Mathematical Applications 56: 3188-3194.

http://dx.doi.org/doi:10.1016/j.camwa.2008.09.013.

9. Das, K. 2012. Influence of thermophoresis and chemical reaction on MHD micropolar fluid flow with variable fluid properties, International Journal Heat Mass Transfer 55: 766-774.

http://dx.doi.org/10.1016/j.ijheatmasstransfer.2012.07.033.

10. Mahmoud, M.A.A. 2007. Thermal radiation effects on MHD flow of a micropolar fluid over a stretching surface with variable thermal conductivity, Physica A 375:401410. http://dx.doi.org/10.1016/j.physa.2006.09.010.

11. Pal, D.; Chatterjee, S. 2011. Mixed convection magnetohydrodynamic heat and mass transfer past a stretching surface in a micropolar fluid-saturated porous medium under the influence of Ohmic heating. Soret and Dufour effects, Communications Nonlinear Science Numerical Simulation 16(3): 1329-1346.

http://dx.doi.org/10.1016/j.cnsns.2010.06.008.

12.Seddeek, M.A.; Odda, S.N.; Akl, M.Y.; Abdelmeguid, M.S. 2009. Analytical solution for the effect of radiation on flow of a magneto-micropolar fluid past a continuously moving plate with suction and blowing, Computational Materials Science 45: 423-428. http://dx.doi.org/10.1016/j.commatsci.2008.11.001.

13. Ishak A.; Nazar, R.; Pop, I. 2009. MHD boundarylayer flow of a micropolar fluid past a wedge with constant wall heat flux, Communications Nonlinear Science Numerical Simulation 14: 109-118. http://dx.doi.org/10.1016/j.cnsns.2007.07.011.

14. Mahmoud, M.A.A.; Waheed, S.E. 2012. MHD stagnation point flow of a micropolar fluid towards a moving surface with radiation, Meccanica 47: 1119-1130. http://dx.doi.org/10.1007/s11012-011-9498-x.

15. Abd-El Aziz, M. 2006. Thermal radiation effects on magnetohydrodynamic mixed convection flow of a micropolar fluid past a continuously moving semi-infinite plate for high temperature differences, Acta Mechanica 187: 113-127. http://dx.doi.org/10.1007/s00707-006-0377-9.

16. Elbarbary, E.M.; Elgazery, N.S. 2005. Flow and heat transfer of a micropolar fluid in an axisymmetric stagnation flow on a cylinder with variable properties and suction, Acta Mechanica 176: 213-229. http://dx.doi.org/10.1007/s00707-004-0205-z.

17. Chamkha, A.J.; Mohamed, R.A.; Ahmed, S.E. 2011. Unsteady MHD natural convection from a heated vertical porous plate in a micropolar fluid with Joule heating, chemical reaction and radiation effects, Meccanica 46: 399-411. http://dx.doi.org/10.1007/s11012-010-9321-0.

18. Alam, M.S.; Rahman, M.M.; Sattar, M.A. 2009. On the effectiveness of viscous dissipation and Joule heating on steady Magnetohydrodynamic heat and mass transfer flow over an inclined radiate isothermal permeable surface in the presence of thermophoresis, Communications Nonlinear Science Numerical Simulation 14: 2132-2143. http://dx.doi.org/10.1016/j.cnsns.2008.06.008. 
19. Tsai, R.; Huang, J.S. 2010. Combined effects of thermophoresis and electrophoresis on particle deposition On to a vertical flat plate from mixed convection flow through a porous medium, Chemical Engineering Journal 157: 52-59. http://dx.doi.org/10.1016/j.cej.2009.10.046.

20. Muhaimin, I.; Kandasamy, R.; Hashim, I. 2009. Thermophoresis and chemical reaction effects on nonDarcy MHD mixed convective heat and mass transfer past a porous wedge in the presence of variable stream Condition, Chemical Engineering Research Design $87: 1527-1535$. http://dx.doi.org/10.1016/j.cherd.2009.04.005.

21. Long Hsiao, K. 2010. Heat and mass transfer for micropolar flow with radiation effect past a nonlinearly stretching sheet, Heat Mass Transfer 46: 413-419. http://dx.doi.org/10.1007/s00231-010-0580-z.

22. Ahmadi, G. 1976. Self similar solution of incompressible micropolar fluid boundary layer over a semi-infinite plate, International Journal Engineering Science 14: 639-646.

Available from internet: http://www.sciencedirect.com/ science/article/pii/0020722576900069.

23. Yacob, N.A.; Ishak, A. 2011. MHD flow of a micropolar fluid towards a vertical permeable plate with prescribed surface heat flux, Chemical Engineering Science Research Design 89: 2291-2297. http://dx.doi.org/10.1016/j.cherd.2011.03.011.
R. Alouaoui, M. N. Bouaziz

\section{INFLUENCE OF THERMOPHORESIS ON MHD MICROPOLAR FLUID OVER MOVED PERMEABLE PLATE}

\section{S u m m a r y}

This work investigates mainly the magnetic field and the thermophoresis effects on the heat and mass transfer in steady laminar boundary layer flow of an incompressible viscous micropolar fluid over a moving vertical and permeable plate subjected to a magnetic field without neglecting the resulting dissipation. The governing differential equations are transformed into the ordinary differential equations which are solved numerically. Results are displayed graphically to illustrate the influence of Magnetic parameter, Thermophoretic parameter, and suction/injection parameter on velocity, microrotation, temperature and species concentration profiles. The rate of mass transfer is affected strongly by Thermophoretic parameterwhile the local skin-friction coefficient and the local wall couple stress are sensitive to the variations of the magnetic field and to the suction case. The micropolarity controls the shear stress but plays a limited role for the others quantities. Heat and mass transfer rate can be regulated principally by the use of a permeable plate with suction.

Keywords: thermophoresis, magnetohydrodynamic, micropolar fluid, moving plate, suction.

Received April 19, 2016 Accepted June 08, 2017 